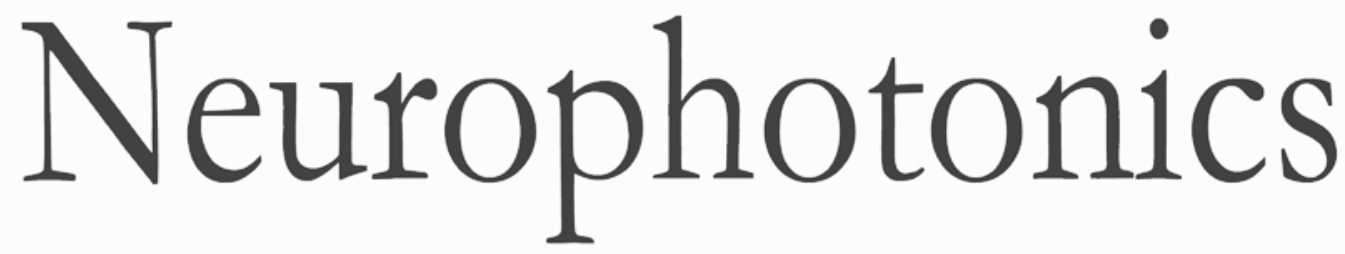

\title{
Proposal of auxiliary diagnosis index for autism spectrum disorder using near-infrared spectroscopy
}

Kazuki Yanagisawa

Nozomi Nakamura

Hitoshi Tsunashima

Naoko Narita 


\title{
Proposal of auxiliary diagnosis index for autism spectrum disorder using near-infrared spectroscopy
}

\author{
Kazuki Yanagisawa, ${ }^{a, *}$ Nozomi Nakamura, ${ }^{b}$ Hitoshi Tsunashima, ${ }^{a}$ and Naoko Narita ${ }^{c}$ \\ ${ }^{a}$ Nihon University, College of Industrial Technology, Department of Mechanical Engineering, 1-2-1 Izumi-cho Narashino-shi, \\ Chiba 275-8575, Japan \\ ${ }^{b}$ Nihon University, Graduate School of College of Industrial Technology, Department of Mechanical Engineering, \\ 1-2-1 Izumi-cho Narashino-shi, Chiba 275-8575, Japan \\ ${ }^{\circ}$ Bunkyo University, Institute of Education, School Education Course, 3337 Minami-Ogishima, Koshigaya, Saitama 343-851 Japan
}

\begin{abstract}
Lack of a diagnostic index is a problem that needs to be overcome in the diagnosis of autism spectrum disorder (ASD), because this problem prevents an objective assessment based on biomarkers. This paper describes the development of a diagnostic index for ASD using near-infrared spectroscopy (NIRS). We investigated continuous prefrontal hemodynamic changes depending on reciprocal disposition of working memory and nonworking memory tasks using two-channel NIRS. NIRS signals in the prefrontal cortex were compared between high-functioning ASD subjects $(n=11)$ and typically developed (TD) subjects $(n=21)$. The brain activities of the TD subjects were related to experimental design. These results were not confirmed in brain activities of ASD subjects, although the task performance rate was almost equivalent. The brain activities of TD subjects and ASD subjects were evaluated using a weighted separability (WS) index obtained from the feature phase of oxy-hemoglobin and its differential value. Calculation of the $t$-test (TD subject versus ASD subject) confirmed that WS was significant. This result showed that the proposed index was useful for evaluation of the brain activity of ASD subjects. ๑ The Authors. Published by SPIE under a Creative Commons Attribution 3.0 Unported License. Distribution or reproduction of this work in whole or in part requires full attribution of the original publication, including its DOI. [DOI: 10.1117/1.NPh.3.3.031413]
\end{abstract}

Keywords: near-infrared spectroscopy; autistic spectrum disorder; auxiliary diagnosis.

Paper 15061SSRR received Dec. 28, 2015; accepted for publication May 17, 2016; published online Jun. 9, 2016.

\section{Introduction}

Developmental disorders is the general term given to developmental delays caused by congenital factors whose characteristics manifest particularly in childhood. However, more recent studies suggest that there should be many other factors, such as epigenetic factors ${ }^{1-3}$ or parenting, ${ }^{4,5}$ which are involved in the establishment of clinical features in the later life of the patients. This makes it difficult to diagnose these disorders simply by the current method of diagnosis, which is conducted by a specialist's medial interview using diagnostic criteria, such as those defined by the American Psychiatric Association in the Diagnostic and Statistical Manual of Mental Disorders 5. However, many disorders in the category of developmental disorders have complex symptoms that make accurate diagnosis difficult, and this difficulty is regarded as a problem.

In previous studies of patients with developmental disorders, Gallagher et al. ${ }^{6}$ used functional magnetic resonance imaging (fMRI) to measure the brain activity of subjects with one type of developmental disorder, autism spectrum disorder (ASD), while the subjects executed different verbal tasks and image recognition tasks. Gallagher et al. reported that activity in the dorsolateral prefrontal cortex was reduced compared to typically developed (TD) subjects. However, because some developmental disorders exhibit characteristics such as hyperactivity and limited interests, it is difficult to obtain accurate measurements using fMRI, which involves the patient being confined and their movements restricted. Therefore, a simpler instrument for measuring brain activity is required.

*Address all correspondence to: Kazuki Yanagisawa, E-mail: kazuki .yanagisawal@nihon-u.ac.jp
One simple method of measuring brain activity is near-infrared spectroscopy (NIRS). In previous studies, Narita et al. ${ }^{7}$ used NIRS to measure changes in blood flow in the frontal lobe of ASD subjects during execution of recognition memory tasks and demonstrated a difference in activity in the dorsolateral prefrontal cortex compared to TD subjects. This study suggested the possibility that NIRS signals could be applicable as a diagnostic indicator for developmental disorders. However, measuring brain activity using NIRS involves problems, such as the fact that the measured values are values of relative change, and that dealing with the measured data is difficult because a standardized analysis method has not been established. These difficulties are serious obstacles in the development of an auxiliary diagnosis index for developmental disorders.

In previous studies relating to an auxiliary diagnosis index using NIRS signals, Yanagisawa et al. ${ }^{8}$ performed neurofeedback on TD subjects using NIRS signals and investigated methods of quantitatively evaluating changes in brain activity characteristics before and after training. Focusing on two feature values, oxy-hemoglobin (oxy-Hb) and the differential value of oxy-Hb, measured using NIRS, Yanagisawa et al. ${ }^{8}$ proposed the use of weighted separability (WS), which can quantitatively evaluate differences in brain activity characteristics during tasks, and demonstrated its effectiveness. Application of this method could enable quantitative evaluation of differences in brain activity patterns between subjects with developmental disorders and TD subjects.

Therefore, for the purpose of developing an auxiliary diagnosis index for ASD from brain activity measurements using NIRS, this study was conducted to reanalyze the previous data of NIRS measurement reported by Narita et al. ${ }^{7}$ to evaluate the NIRS signals of TD subjects and ASD subjects during 
execution of recognition memory tasks using the WS index developed by Yanagisawa et al. ${ }^{8}$ and it investigated an NIRSbased auxiliary diagnosis index for developmental disorders.

\section{Description of the Experiment}

\subsection{Experimental Subjects}

The subjects and data for this study were basically overlapped with those previously reported by Narita et al., ${ }^{7}$ except for one TD subject who was omitted because of data incompletion. Briefly, 11 ASD subjects [intelligence quotient (IQ) $>65$ by Wechsler Intelligence Scale for Children-III (WISC-III) or Wechsler Adult Intelligence Scale III (WAIS-III); aged 14 to 46 years, mean: 29.5 years; 3 males, 8 females; all right-handed] and 21 healthy controls (aged 19 to 51 years, mean: 25.3 years; 5 male, 16 females; 20 right-handed and 2 left-handed) were studied.

All subjects had been diagnosed and assessed at Ushiku Aiwa Hospital (Ibaraki, Japan) by pediatric and adult neurology and psychology specialists (including Narita) on the basis of developmental history and current presentation, according to the Diagnostic and Statistical Manual of Mental Disorders IV and the Japanese version of the Autism Diagnostic InterviewRevised as described elsewhere. ${ }^{7}$ Among the 11 ASD subjects, 9 were diagnosed with Asperger syndrome and 2 with highfunctioning autistic syndrome. Mean full scale IQ was 99.4 (range 73 to 118), mean verbal IQ was 107.6 (range 89 to 120), and mean performance IQ was 89.5 (range 54 to 118 ) among the ASD subjects assessed by WISC-III or WAIS-III. All ASD subjects were free from any antipsychotic drugs or psychotherapy when participating in this study. This study was approved by the Research and Ethical Committee of Bunkyo University, and written informed consent was obtained from each subject or their legal guardian prior to the study (Approved No. 20-001).

\subsection{Experimental Method and Measuring Conditions}

ASD subjects are considered to perform poorly in working memory (WM) tasks involving short-term memory retention, and recent studies have identified disordered activity in the dorsolateral prefrontal cortex during WM tasks as well as difficulty in switching between tasks. ${ }^{9-11}$ Therefore, to measure brain activity associated with switching between recognition memory tasks, Narita et al. ${ }^{7}$ developed the exercise shown in Fig. 1, which involves switching successively between two activities: a WM task and a control nonworking memory (NWM) task that does not require short-term memory retention.

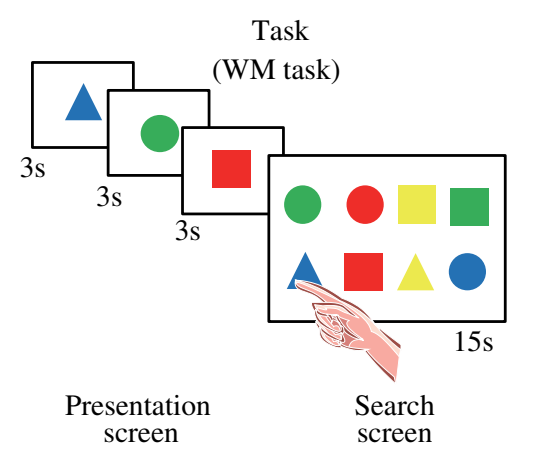

\section{Control (NWM task)}

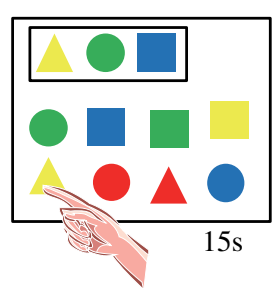

Fig. 1 Experimental problem.
In the WM task, subjects were instructed to memorize graphics of different color (four colors: red, blue, green, and yellow) and shape (three shapes: round, triangular, and square), which were presented successively as stimuli every $3 \mathrm{~s}$ on a computer screen (presentation screen), and then, from a screen showing eight graphics (search screen), to find and point a finger at the graphics presented earlier in the same order as they were presented.

In the control, subjects were instructed to look at a screen showing eight graphics and to find and point a finger at the graphics in the same order as the graphics (presentation graphics) shown in a box at the top of the same screen.

This task and control were considered to be one run of the experiment, and the experiment was run six times for each subject. In the first run, there was one graphic for memorization in the task and one graphic presented in the control, and these were each increased by one graphic in each run, so that the number of graphics in the sixth run was six each.

In the WM task, the graphics presentation screen was shown for 3 to $18 \mathrm{~s}$, followed by $15 \mathrm{~s}$ search screen. Graphics presentation time of each WM task was encompassed by two guidance screens, which appeared for $3 \mathrm{~s}$ each. The presentation time for the NWM task was $15 \mathrm{~s}$.

In this study, brain activity during the recognition memory task was measured using the near-infrared oxygenation monitor NIRO 200, manufactured by Hamamatsu Photonics K.K. Brain activity was measured using two measurement channels. The NIRO 200 (Hamamatsu Photonics, Tokyo, Japan) uses laser-emitting diodes to generate light at three different wavelengths $(775,810$, and $850 \mathrm{~nm})$. It employs the technique of spatial-resolved spectroscopy and uses multiple closely spaced detectors to measure light attenuation as a function of sourcedetector separation. The source-detector distance is $30 \mathrm{~mm}$. The measurement positions were Fp1 and Fp2 in the dorsolateral prefrontal cortex according to the International 10-20 System.

\subsection{Experimental Results}

The NIRS signals also include signals other than brain activity related to the task, and so it is necessary to extract brain activity related to the task. By performing a multiresolution analysis of the measured NIRS signals using a discrete wavelet transform, the noise of the measuring equipment, the influence of breathing and heartbeat fluctuation, Mayer waves, and so on are removed, and components related to the task are extracted. In this study, one run of the experiment took 39 to $54 \mathrm{~s}$, and so fluctuations related to the task with this period as the central component ( $\sim 16$ to $256 \mathrm{~s}$ ) were extracted as brain activity. $^{12,13}$

Figures 2-4 show particularly representative fluctuations in NIRS signals from among the subjects. Figure 2 shows the results of one TD subject, and Figs. 3 and 4 show the results of one ASD subject. In the NIRS signals of the TD subject in Fig. 2, a tendency for oxy-Hb to increase during the WM task and decrease during the NWM task can be identified in all runs of the experiment. This shows that the brain is stimulated during the WM task.

In the ASD subjects, two patterns that differed from the TD subjects were identified. One was that the signals were not reproducible and indicated asynchronous brain activity with no relation to whether the task was WM or NWM, as shown in Fig. 3. The second pattern was of oxy-Hb decreasing during 

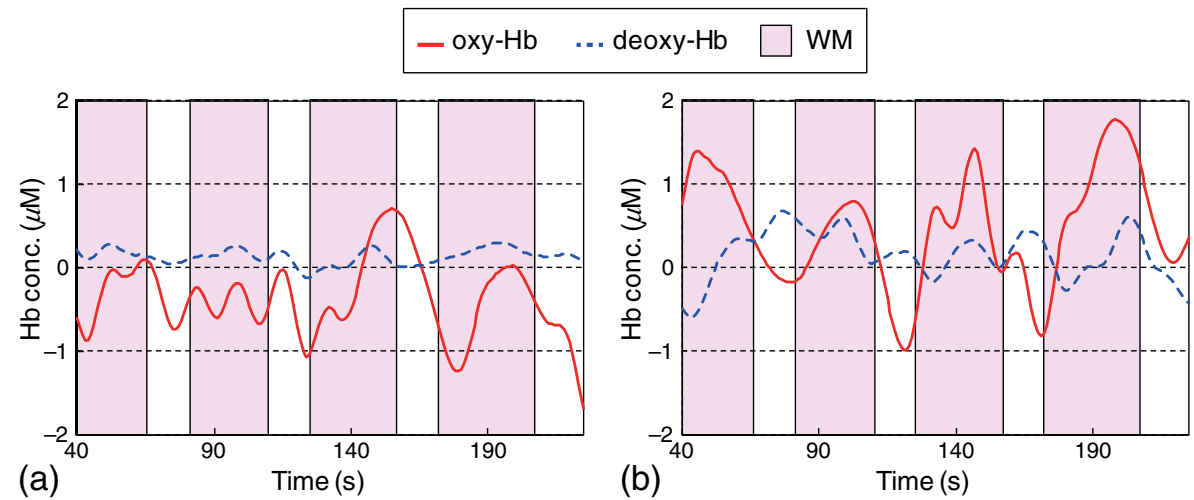

Fig. 2 NIRS signal of TD subjects (left outer portion 2ch): (a) TD subject1 and (b) TD subject2.

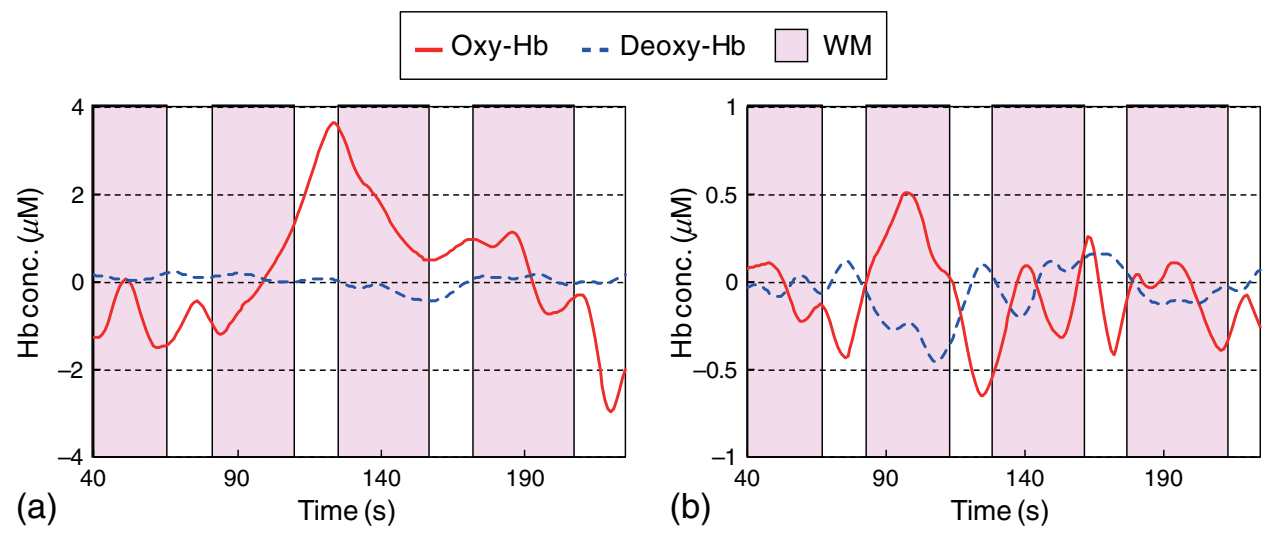

Fig. 3 Asynchronous NIRS signal of ASD1 subjects (left outer portion 2ch): (a) ASD subject1 and (b) ASD subject2.

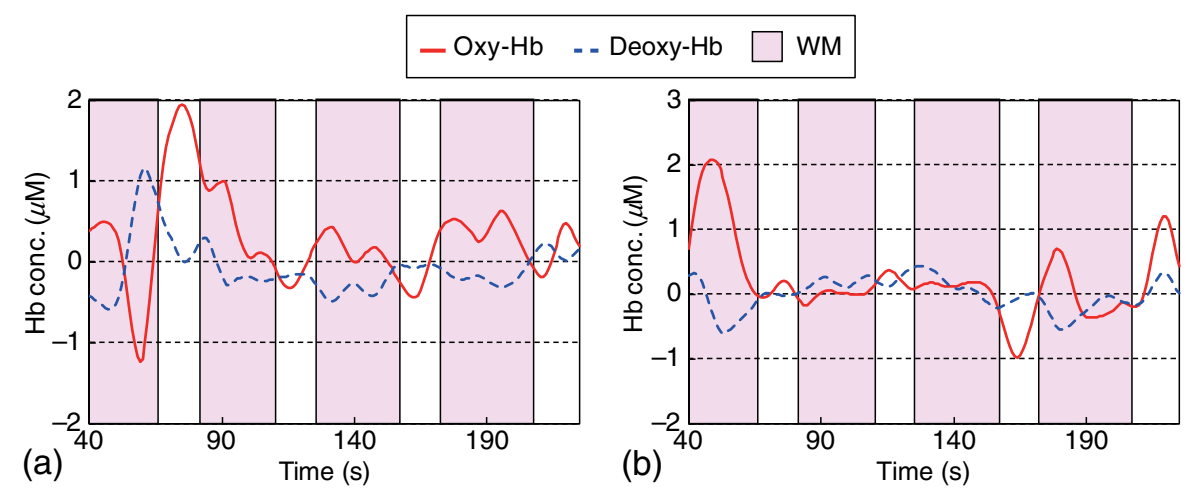

Fig. 4 Opposite pattern of fluctuation to TD subjects (left outer portion 2ch): (a) ASD subject3 and (b) ASD subject4.

the WM task and increasing during the NWM task, which is the opposite pattern of fluctuation to TD subjects, as shown in Fig. 4.

Our experiment succeeded in identifying differences between the NIRS signals of TD subjects and ASD subjects, as shown in Figs. 2-4. However, it is difficult to make comparisons between the experimental subjects using NIRS signals, which are values of relative change, and quantitative evaluation is not possible. Therefore, an index for quantitatively evaluating NIRS signals is required for application as an auxiliary diagnosis index for developmental disorders.

\section{Evaluation Index}

\section{1 $\mathrm{Oxy}-\mathrm{Hb}$ and the Differential Value of $\mathrm{Oxy}-\mathrm{Hb}$}

This study focuses on oxy-Hb and the differential value of oxy$\mathrm{Hb}$ to quantitatively evaluate NIRS signals. Figure 5(a) shows typical fluctuations of TD subjects in oxy-Hb and the differential value during the task. Oxy-Hb increases in association with the task, and the differential value of oxy-Hb, which is its rate of change, increases sooner than oxy-Hb. ${ }^{12,13}$ Figure 5(b) shows the phase plane created by plotting oxy-Hb on the horizontal axis and the differential value of oxy-Hb on the vertical axis. 


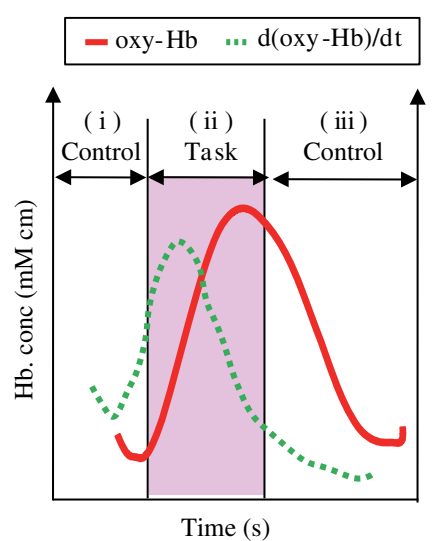

(a)

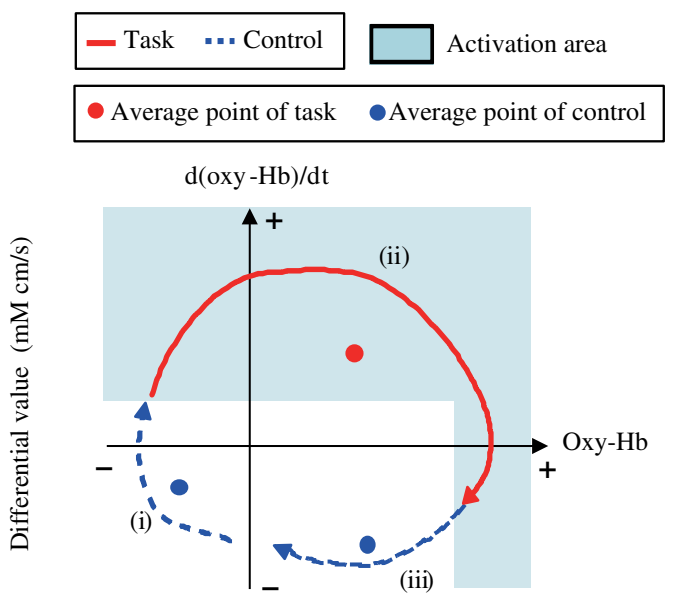

(b)

Fig. 5 Phase plane of oxy- $\mathrm{Hb}$ and differential value (TD subjects): (a) change of oxy-Hb and differential value and (b) phase plane of oxy- $\mathrm{Hb}$ and differential value.

The brain can be regarded as "active" when the value of oxy$\mathrm{Hb}$ is high, or when the value of oxy-Hb is increasing, ${ }^{13}$ that is, the differential value of oxy-Hb is positively high even if the value of oxy-Hb is low. Therefore, we analyzed the oxy-Hb trajectory within the shaded portion of Fig. 5(b) as an indicator of task-related brain activation.

Focusing on the average point of the trajectory for task/control, respectively, on this phase plane, the average point during the task appears in the first quadrant, where oxy- $\mathrm{Hb}$ and the differential value of oxy-Hb are high, and the average point during the control appears in the third quadrant, where both oxy-Hb and the differential value are low, as shown in Fig. 6.

In the WM task performed in this experiment, it was confirmed that the dorsolateral prefrontal cortex is active during the task, and so oxy-Hb is thought to increase and the brain becomes active during the WM task. Therefore, the average point during the WM task appears in the first quadrant of the phase plane. In the NWM task, because it is a copying task

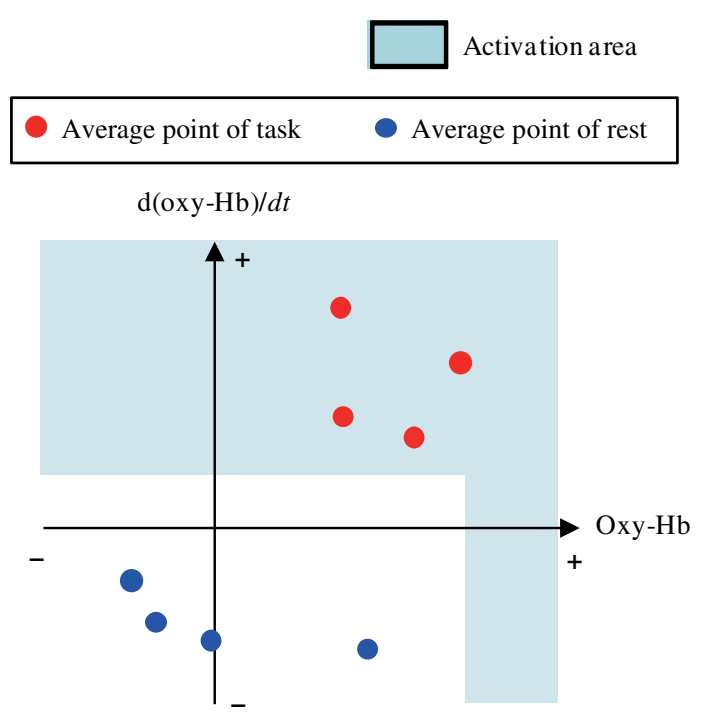

Fig. 6 Distribution of the phase plane of the task and the control pattern diagram. that does not require short-term memory, oxy-Hb is thought to decrease and the brain returns to its resting state. Therefore, as during the control, the average point appears in the third quadrant on the phase plane.

\subsection{Method of Evaluating Near-Infrared Spectroscopy Signal in Regard to Separability}

A quantitative evaluation using the separability index was performed based on the distribution of average points for task/control in the phase plane created from oxy- $\mathrm{Hb}$ and the differential value of oxy-Hb. Separability is an index that evaluates the cohesiveness of signals within a phase plane, and it can evaluate differences in signal characteristics in respective tasks.

Separability is expressed as a ratio of within-class variance and between-class variance, and in this study, it was used to evaluate brain activity characteristics based on the distribution of average points for task/control in the phase plane. First, the mean value of measured oxy- $\mathrm{Hb}$ in one task or control was taken as $p_{\text {oxy }}$, and the mean value of the differential value of oxy-Hb as $\dot{p}_{\text {oxy }}$. Representing these as an average point in the phase plane, $\mathbf{p}$,

$\mathbf{p}=\left(p_{\text {oxy }}, \dot{p}_{\text {oxy }}\right)$.

The set of average points belonging to a task group was taken as $X_{\text {task }}$, the number of average points included in $X_{\text {task }}$ as $n_{\text {task }}$, the mean of all tasks as $\mathbf{m}_{\text {task }}$, the set of average points belonging to the control group as $X_{\text {control }}$, the number of average points included in $X_{\text {control }}$ as $n_{\text {control, }}$, and the mean of all controls as $\mathbf{m}_{\text {control }}$. The total number of average points was taken as $n$ and the mean of all tasks/controls as $\mathbf{m}$. Expressing withinclass variance as $\sigma_{W}^{2}$, and between-class variance as $\sigma_{B}^{2}$ :

$$
\begin{aligned}
\sigma_{W}^{2}= & \frac{1}{n}\left\{\sum_{p \in X_{\text {task }}}\left(\mathbf{p}-\mathbf{m}_{\text {task }}\right)^{T}\left(\mathbf{p}-\mathbf{m}_{\text {task }}\right)\right. \\
& \left.+\sum_{p \in X_{\text {control }}}\left(\mathbf{p}-\mathbf{m}_{\text {control }}\right)^{T}\left(\mathbf{p}-\mathbf{m}_{\text {control }}\right)\right\},
\end{aligned}
$$



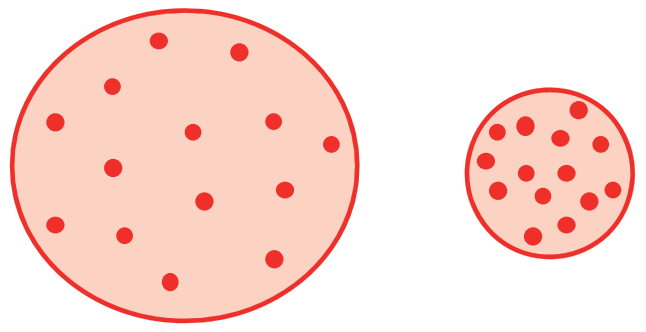

Fig. 7 Example of the variance of the average points in the same group.

$$
\begin{aligned}
\sigma_{B}^{2}= & \frac{1}{n}\left\{n_{\text {task }}\left(\mathbf{m}_{\text {task }}-\mathbf{m}\right)^{T}\left(\mathbf{m}_{\text {task }}-\mathbf{m}\right)\right. \\
& \left.+n_{\text {control }}\left(\mathbf{m}_{\text {control }}-\mathbf{m}\right)^{T}\left(\mathbf{m}_{\text {control }}-\mathbf{m}\right)\right\},
\end{aligned}
$$

Separability can be defined using Eqs. (2) and (3) as

$$
J_{\sigma}=\frac{\sigma_{B}^{2}}{\sigma_{W}^{2}} .
$$

Within-class variance from Eq. (2) represents the variance of the average points in the same group. ${ }^{14,15}$ When the average points of a group are widely distributed (left side of Fig. 7), $\sigma_{W}^{2}$ is high, whereas, when the average points of a group are narrowly distributed (right side of Fig. 7), $\sigma_{W}^{2}$ is low.

Between-class variance from Eq. (3) represents the variance of the centroids of groups. ${ }^{14,15}$ When the average points for task/ control are distributed separately (left side of Fig. 8), $\sigma_{B}^{2}$ is high, whereas, when the average points for task and control are intermingled (right side of Fig. 8), $\sigma_{B}^{2}$ is low.

The smaller the within-class variance and the larger the between-class variance, the greater the difference between characteristics during the task and the control. Basically, when the average points for task and control are intermingled and widely distributed, the separability value is low, whereas, when the average points for task/control are distributed separately and their distributions are narrow with the average points close together, the separability value is high.

\subsection{Problems of Separability and Proposal of Weighted Separability}

The brain activity of TD subjects shows an increase in oxy-HB during a WM task and a decrease to return to the baseline during an NWM task. This means that focusing on the average point of trajectories for WM and NWM tasks, respectively, in the phase
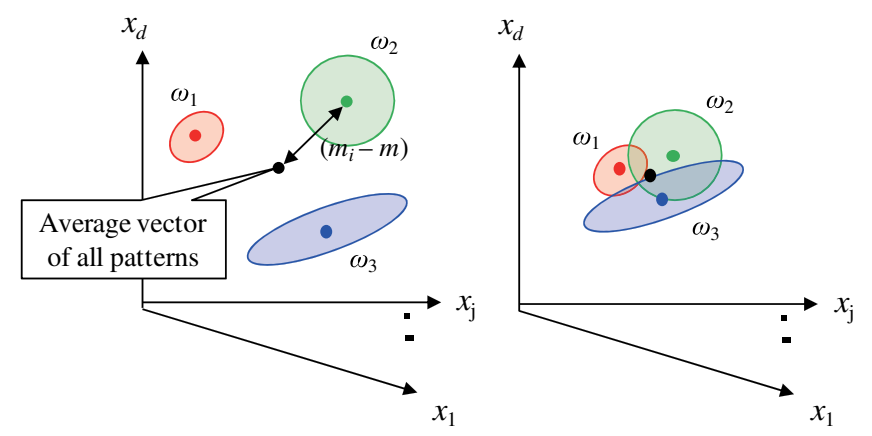

Fig. 8 Example of the variance of the centroids of groups. plane, the average point during the WM task appears in the first quadrant, where oxy-Hb and the differential value of oxy-Hb both have large values, and the average point during the NWM task appears in the third quadrant, where both values are small. If the WM and NWM tasks each show high reproducibility and clear characteristics, then the separability value is high.

The brain activity of ASD subjects shows (i) disordered and asynchronous brain activity with no relation to the task or (ii) a decrease in oxy-Hb during the WM task and an increase during the NWM task, which is the opposite behavior to TD subjects. In regard to brain activity like (i), the average points for WM and NWM tasks appear intermingled on the phase plane, and so the respective characteristics are unclear and the value of separability is low. In regard to brain activity like (ii), the average points for the WM task appear in the third quadrant and the average points for the NWM task appear in the first quadrant. This result is thought to reflect a delay in brain activity due to the difficulty that ASD subjects have in switching between tasks.

However, because separability evaluates only the cohesiveness of signals, the value of separability is high whether the average points for the WM task appear in the first quadrant and the average points for the NWM task appear in the third quadrant, like a TD subject, or the average points for the WM task appear in the third quadrant and the average points for the NWM task appear in the first quadrant, like an ASD subject exhibiting (ii). It is, therefore, difficult to evaluate the characteristic differences between TD subjects and ASD subjects exhibiting fluctuations like (ii).

Therefore, this study focused on the WS $J_{\sigma}^{\prime}$ developed by Yanagisawa et al. ${ }^{8}$ The implications of NIRS signals are very different when oxy-Hb increases during the task and returns to baseline in the control, compared to when oxy-Hb decreases during the task and increases in the control, and so Yanagisawa et al. ${ }^{8}$ developed a WS for weighting quadrants of the phase plane. WS $J_{\sigma}^{\prime}$ is defined by the following equation, where the weighting factor is determined based on how many average points for task/control exist in each quadrant:

$J_{\sigma}^{\prime}=\frac{i+1}{j+1} J_{\sigma}$.

In this case, $i$ is the sum of the number of task average points in the first quadrant and the number of control average points in the third quadrant. Conversely, $j$ is the sum of the number of task average points in the third quadrant and the number of control average points in the first quadrant. $i$ increases when there is typical brain activity, in which the brain activity is stimulated during the task and returns to the baseline during the control. $j$ increases when there is fluctuation such that oxy- $\mathrm{Hb}$ decreases during the task and increases during the control.

Therefore, WS is larger when the pattern of brain activity is that of a TD subject, in which case $n$ increases and $m$ decreases. Conversely, WS is smaller in the case of ASD subjects, where $n$ decreases and $m$ increases.

\section{Evaluation Results}

\subsection{Task Performance}

The mean task performance rate was calculated among the subject groups. Tables 1 and 2 show the performance rates of TD and ASD subjects. The average task performance rate of WM 
Table 1 Performance rate of TD subject $(n=21)$

\begin{tabular}{lccc} 
Condition & Performance rate (\%) & Condition & Performance rate (\%) \\
\hline 1WM & 100 & 1NWM & 100 \\
2WM & 100 & 2NWM & 100 \\
3WM & 76.1 & 3NWM & 98.4 \\
4WM & 63.1 & $4 N W M$ & 100 \\
5WM & 55.7 & $5 \mathrm{NWM}$ & 100 \\
$6 \mathrm{WM}$ & 27.8 & $6 \mathrm{NWM}$ & 92.1 \\
Average & 70.5 & Average & 98.4 \\
\hline
\end{tabular}

Table 2 Performance rate of ASD subject $(n=11)$.

\begin{tabular}{lccc} 
Condition & Performance rate (\%) & Condition & Performance rate (\%) \\
\hline 1WM & 100 & 1NWM & 100 \\
2WM & 100 & 2NWM & 100 \\
3WM & 78.8 & 3NWM & 100 \\
4WM & 56.8 & 4 NWM & 100 \\
5WM & 61.8 & $5 N W M$ & 100 \\
$6 W M$ & 27.3 & $6 N W M$ & 100 \\
Average & 70.8 & Average & 100 \\
\hline
\end{tabular}

was $70.5 \%$ in TD and $70.8 \%$ in ASD, whereas that of NWM was $98.4 \%$ in TD and $100 \%$ in ASD. Both TD and ASD subjects showed a gradual decrease in the task performance rate with increasing task block number during the WM task in contrast to that during the NWM task, which was almost $100 \%$ for each task block.

\subsection{Phase Plane and Weighted Separability}

Using the phase plane created from oxy-Hb and the differential value of oxy-Hb, the characteristics of NIRS signals during
WM/NWM tasks were evaluated using WS, an index for quantitatively evaluating NIRS signal characteristics.

In the first run of the experiment for each subject, the WM task was very easy with just one graphic for memorization, whereas in the sixth run, the level of difficulty of the task was high with six graphics for memorization. An evaluation conducted previously by Narita et al. ${ }^{7}$ did not succeed in identifying a significant difference in the first and sixth runs. This is considered to be due to the fact that, when there was one graphic for memorization, the task was extremely easy and so no stimulation was shown in relation to the task; when there were six graphics for memorization, the task was extremely difficult, and some subjects gave up engaging with the task. Therefore, in this study, brain activity in the first and sixth runs was excluded when calculating WS.

Figure 9 shows phase planes and WS values for TD and ASD subjects. Figure 9(a) shows the results for a TD subject, Fig. 9(b) shows the results for an ASD subject who did not demonstrate activity related to the task, and Fig. 9(c) shows results indicating an opposite fluctuation pattern to that of a TD subject.

In the phase plane for a TD subject in Fig. 9(a), the average points during the WM task are distributed in the first quadrant and the average points during the NWM task in the third quadrant, and the WM task and NWM task characteristics are clear. This indicates that brain activity was stimulated during the WM task and returned to the baseline during the NWM task, which is typical change during a cognitive task. Therefore, the WS value was high, at 5.06 .

However, in the ASD results in Fig. 9(b), the average points for the WM/NWM tasks are intermingled in the phase plane, which confirms that the characteristics for each task are unclear. Therefore, the WS value was low at 0.0461 . Moreover, looking at the results shown in Fig. 9(c), for an ASD subject who demonstrated fluctuations that were opposite to a TD subject, the average points during the NWM task are distributed mainly in the first quadrant and the average points during the WM task mainly in the third quadrant, and because differences in characteristics between the tasks can be identified, it is not possible to evaluate differences from TD subjects using separability. However, WS is low at 0.664 , and it was possible to evaluate that the brain activity pattern differs from TD subjects by using WS.

Furthermore, Fig. 10 shows the results of a $t$-test carried out on the difference in WS between the 21 TD subjects and the 11 ASD subjects. In the right dorsolateral prefrontal cortex, WS

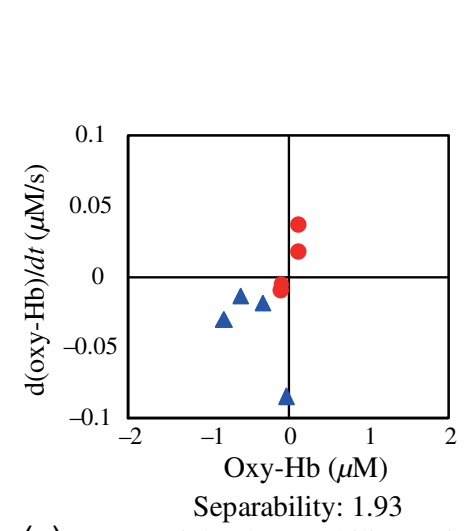

(a)

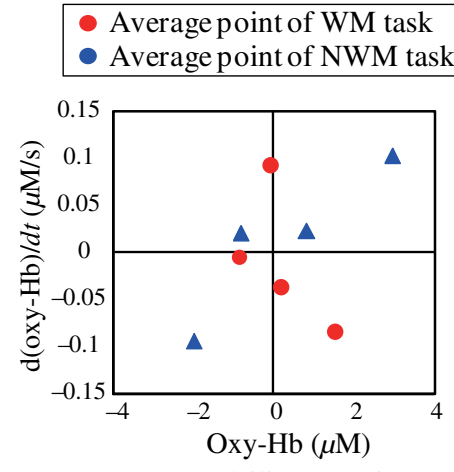

Separab ility: 0.0461

(b) Weighted separability: $0.0230 \quad$ (c)

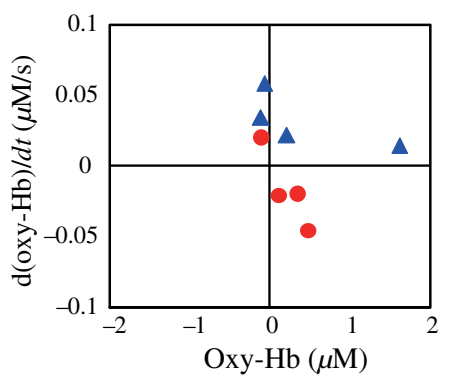

Separability: 1.99

Weighted separability: 0.664

Fig. 9 Phase plane and WS: (a) TD subject, (b) ASD subject1, and (c) ASD subject2. 

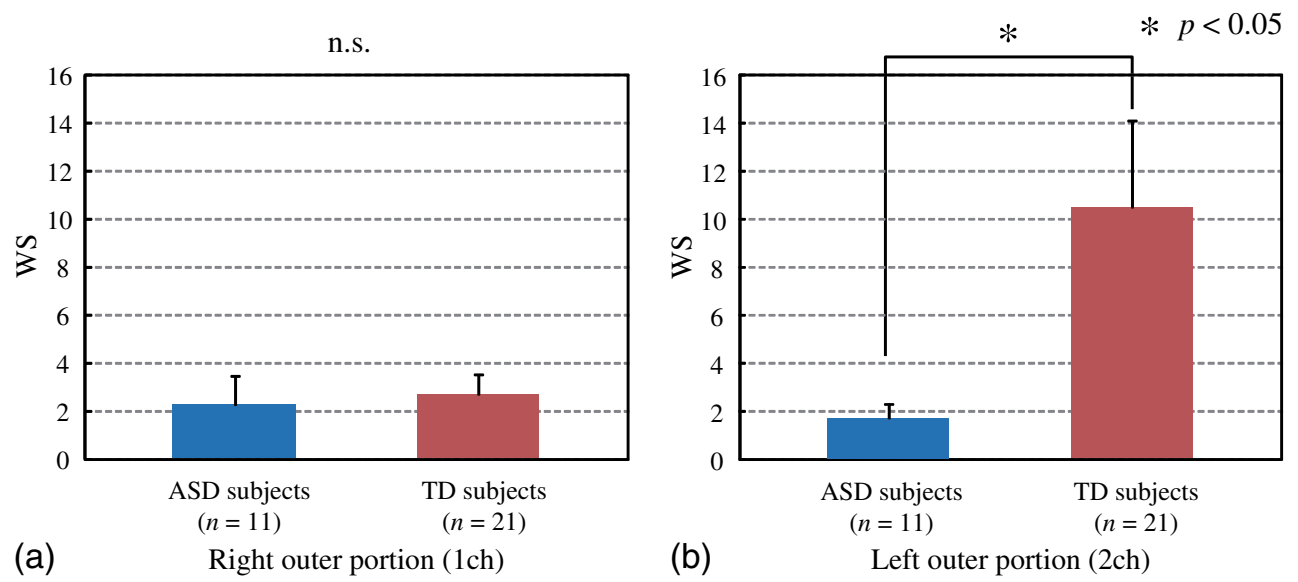

Fig. 10 Result of $t$-test.

was low for both TD and ASD subjects, and a significant difference could not be identified. In the left dorsolateral prefrontal cortex, the brains of TD subjects were activated in relation to the WM task, and so the WS value was high, whereas, ASD subjects who demonstrated a different activity pattern to TD subjects had a low WS value, and it was possible to confirm that this difference was significant.

\subsection{Discussion}

We succeeded in confirming a difference in brain activity between TD subjects and ASD subjects in the left dorsolateral prefrontal cortex through evaluation using WS. The previous research by Narita et al. $^{7}$ reported a difference in activity between TD subjects and ASD subjects in the left dorsolateral prefrontal cortex with regard to mean oxy-Hb values during WM tasks. However, the evaluations using mean oxy-Hb values did not evaluate the difficulty that ASD subjects have in switching between tasks, and it was therefore necessary to evaluate successive changes in brain activity from a WM task to an NWM task.

In the evaluation carried out in this study using WS, it was possible to evaluate changes in response to a block design in which the brain was active during a WM task and brain activity returned to baseline during an NWM task to evaluate successive changes in NIRS signals. In this study, ASD subjects had lower WS values for the left dorsolateral prefrontal cortex compared to TD subjects. This result reflects the fact that successive changes in brain activity associated with switching tasks are inefficient fluctuations in the case of ASD subjects in comparison with TD subjects, and it demonstrates the validity of using WS as an auxiliary diagnosis index, as proposed in this study.

The previous research using fMRI has reported that ASD subjects exhibit reduced activity in the left dorsolateral prefrontal cortex, considered to be the region for responsibility, during execution of a "theory of mind" task compared to TD subjects, ${ }^{16,17}$ and other previous research has identified a relationship between brain activity in the left dorsolateral area and emotional control. ${ }^{18}$ Koshino et al. ${ }^{10}$ also used fMRI to measure changes in blood flow in the frontal lobe of ASD subjects during WM tasks and demonstrated a difference in activity in the prefrontal cortex compared to TD subjects.

Therefore, differences between ASD subjects and TD subjects can be expected to appear in brain activity in the left dorsolateral prefrontal cortex, and it is considered valid to focus on activity in the left dorsolateral area in developing an auxiliary diagnosis index.

The weaknesses of the proposed method are that it is necessary to set a block design experiment. In future work, as an alternative to WS, we will develop an auxiliary diagnosis index using NIRS signal of resting state.

\section{Summary}

In this study, to develop a diagnostic aid for ASD using NIRS, we evaluated NIRS signals of TD subjects and ASD subjects during execution of cognitive tasks using WS, an index developed by Yanagisawa et al. $^{8}$ that is capable of quantitatively evaluating the characteristics of NIRS signals during task/ control.

Focusing on the difficulty ASD subjects have in switching tasks, we set up a task that involved successively performing a WM task and an NWM task. Using phase planes created from oxy- $\mathrm{Hb}$ and the differential value of oxy- $\mathrm{Hb}$ for brain activity in TD subjects and ASD subjects during that task, we investigated the validity of using the WS index, which evaluates characteristics of brain activity, as an auxiliary diagnosis index for ASD subjects.

We succeeded in confirming that the brains of most TD subjects were active during the WM task and returned to the baseline during the NWM task. Therefore, WS values were high. In the ASD subjects, we succeeded in confirming asynchronous brain activity regardless of the task and an activity pattern in which fluctuations related to the task were greatly delayed so that the brain was active during the NWM task, which is the opposite of TD subjects. WS values were low. As a result of conducting a $t$-test on WS for TD subjects and ASD subjects (21 TD subjects and 11 ASD subjects), we were able to detect a significant difference in the left dorsolateral prefrontal cortex.

The previous research ${ }^{7}$ was not able to evaluate successive changes in brain activity associated with the difficulty ASD subjects have in switching between tasks, but the use of WS makes it possible to evaluate successive changes in NIRS signals, and we succeeded in quantitatively evaluating the differences in brain activity characteristics of TD subjects and ASD subjects. The abovementioned results indicate the possibility of using a method of evaluating brain activity measured by NIRS using WS as an auxiliary diagnosis index for ASD.

Hereafter, focusing on neurofeedback training for ASD subjects, which is a technique in which the subject controls their 
brain activity using their own will, we intend to investigate changes in WS and changes in clinical symptoms as a result of training.

\section{Acknowledgments}

The authors feel deep gratitude for all the subjects who volunteered for this study.

\section{References}

1. E. R. Berko and J. M. Greally, "How might epigenetic dysregulation in early embryonic life contribute to autism spectrum disorder?," Epigenomics 7(1), 1-4 (2015).

2. T. Ohkawara et al., "Maternal viral infection during pregnancy impairs development of fetal serotonergic neurons," Brain Dev. 37, 88-93 (2015).

3. M. Narita et al., "Nonexploratory movement and behavioral alterations in a thalidomide or valproic acid-induced autism model rat," Neurosci. Res. 66, 2-6 (2010).

4. G. Kochanska et al., "Developmental interplay between children's biobehavioral risk and the parenting environment from toddler to early school age: prediction of socialization outcomes in preadolescence," Dev. Psychopathol. 27(3), 775-790 (2015).

5. J. Belsky, M. J. Bakermans-Kranenburg, and M. H. van IJzendoorn, "For better and for worse. Differential susceptibility to environmental influences," Curr. Dir. Psychol. Sci. 16(6), 300-304 (2007).

6. H. L. Gallagher et al., "Reading the mind in cartoons and stories: an fMRI study of 'theory of mind' in verbal and nonverbal tasks," Neuropsychologia 38, 11-21 (2000).

7. N. Narita et al., "Impaired prefrontal cortical response by switching stimuli in autism spectrum disorders," J. Paediatr. Neurol. 10(2), 87-94 (2012).

8. K. Yanagisawa, H. Tsunashima, and K. Sakatani, "Proposal of evaluation method for NIRS signal in neurofeedback training for frontal cortex," Jpn. J. Ergon. 51(1), 42-51 (2015) (in Japanese).

9. S. D. Steele et al., "Spatial working memory deficits in autism," J. Autism Dev. Disord. 37(4), 605-612, (2007).

10. H. Koshino et al., "Functional connectivity in an fMRI working memory task in high-functioning autism," Neuroimage 24(3), 810-821 (2005).

11. G. Stoet and B. Lopez, "Task-switching abilities in children with autism spectrum disorder," Eur. J. Dev. Psychol. 8(1), 244-260 (2011).
12. H. Tsunashima, K. Yanagisawa, and M. Iwadate, "Measurement of brain function using near-infrared spectroscopy (NIRS)," in Neuroimaging-Methods, pp. 75-98, InTech, Rijeka, Croatia (2012).

13. H. Tsunashima and K. Yanagisawa, "Measurement of brain function of car driver using functional near-infrared spectroscopy (fNIRS)," Comput. Intell. Neurosci. 2009, 1-12 (2009).

14. S. Ozawa, Y. Sakaguchi, and M. Kotani, "A study of feature extraction using supervised independent component analysis," in Proc. of the Int. Joint Conf. on Neural Networks, Vol. 4, pp. 2958-2963 (2001).

15. K. J. Cios, Data Mining: A Knowledge Discovery Approach, Springer, New York (2007).

16. P. Fletcher, "Other minds in the brain: a functional imaging study of "theory of mind" in story comprehension," Cognition 57(2), 109-128 (1995).

17. F. Happé et al., "'Theory of mind' in the brain. Evidence from a PET scan study of Asperger syndrome," NeuroReport 8(1), 197-201 (1996).

18. E. K. Miller and J. D. Cohen, "An integrative theory of prefrontal cortex function," Аnпи. Rev. Neurosci. 24, 167-202 (2001).

Kazuki Yanagisawa received his BS and MS degrees in physics from Nihon University in 2008 and 2010, respectively, and his PhD in engineering from Nihon University in 2013. He is a research assistant at the Nihon University.

Nozomi Nakamura: Biography is not available.

Hitoshi Tsunashima is a professor and the head of Department of Mechanical Engineering, College of Industrial Technology, Nihon University. His main research expertise includes condition monitoring of railway, multibody dynamics, human factors, and ergonomics. His major research activities are condition monitoring of railway system, application of multiple model approach for vehicle state estimation and control, measurement of brain function using NIRS, brain-computer interface $(\mathrm{BCl})$ using NIRS.

Naoko Narita is a professor in the Education Department of Bunkyo University, and is also a pediatrician who specialized in pediatric psychology and neurology. Her main research expertise includes biological and physiological etiology of autism spectrum disorders, and developmental neurology of typically-developed children. Her major research activities include clinical study of autism spectrum disorders and measurement of brain function using NIRS and $\mathrm{BCl}$ using NIRS. 